\title{
GPLSIUA: Combining Temporal Information and Topic Modeling for Cross-Document Event Ordering
}

\author{
Borja Navarro-Colorado and Estela Saquete \\ Natural Language Processing Group \\ University of Alicante \\ Alicante, Spain \\ borja@dlsi.ua.es, stela@dlsi.ua.es
}

\begin{abstract}
Building unified timelines from a collection of written news articles requires cross-document event coreference resolution and temporal relation extraction. In this paper we present an approach event coreference resolution according to: a) similar temporal information, and b) similar semantic arguments. Temporal information is detected using an automatic temporal information system (TIPSem), while semantic information is represented by means of LDA Topic Modeling. The evaluation of our approach shows that it obtains the highest Micro-average F-score results in the SemEval2015 Task 4: "TimeLine: Cross-Document Event Ordering" (25.36\% for TrackB, $23.15 \%$ for SubtrackB), with an improvement of up to $6 \%$ in comparison to the other systems. However, our experiment also showed some drawbacks in the Topic Modeling approach that degrades performance of the system.
\end{abstract}

\section{Introduction}

Since access to knowledge is crucial in any domain, connecting and time-ordering the information extracted from different documents is a very important task. The goal of this paper is therefore to build ordered timelines for a set of events related to a target entity. In doing so, our approach is dealing with two problems: a) cross-document event coreference resolution and b) cross-document temporal relation extraction.

In order to arrange event mentions in a timeline it is necessary to know which event mentions co-refer to the same event or fact and occur at the same moment. Our approach attempts to formalize the idea that two or more event mentions co-refer if they have not only temporal compatibility (the events occur at the same time) but also semantic compatibility (the event mentions refers to the same facts, location, entities, etc.).

Of a set of event mentions in one or more texts, our proposal groups together the event mentions that (i) have the same or a similar temporal reference, (ii) have the same or a similar event head word, and (iii) whose main arguments refer to the same or similar topics. In order to evaluate the system, we have participated in the SemEval-2015 Task 4 "TimeLine: Cross-Document Event Ordering".

In the following sections we will present the theoretical background to our approach (section 2) and the main technical aspects (sections 3 and 4). Then we will present the results obtained (section 5) and some conclusions.

\section{Background}

Two or more event mentions co-refer when they refer to the same real fact or event. Two events can denote the same fact whereas the linguistic mentions have a different syntax structure, different words, or even a different meaning. Whatever the case may be, both event mentions must be semantically related.

An event mention is formed of an event head (usually a verb or a deverbal noun) that is related to a semantic structure (linguistically represented as an argument structure with an agent, patient, theme, instrument, etc., that is, the semantic roles) in which there are some event participants (entities) 
and which is located in place and time (Levin and Rappaport-Hovav, 2005; Hovav et al., 2010). The meaning of an event mention is therefore not only the meaning of the event head, but also the compositional meaning of all the components and their relations: head, participants, time, place, etc.

In order to detect this semantic relation between event mentions, previous papers have isolated the main components of the event structure. For instance, Cybulska and Vossen (2013) apply an event model based on four components: location, time, participant and action. Moreover, with regard to temporal information, only explicit temporal expressions that appears in the text are considered, but no temporal information is inferred by navigating temporal links. Bejan and Harabagiu (2014) use a rich set of linguistic features to model the event structure, including lexical features such as head word and lemmas, class features such as PoS or event class, semantic features such as WordNet sense or semantic roles frames, etc. They use an unsupervised approach based on a non-parametrical Bayesian model.

\section{Our Approach}

In our approach we represent each event mention as a head word (the event tag in the TimeML (Saurí et al., 2006) annotation scheme) related to a temporal expression (implicit or explicit), a set of entities (0 or more), and a set of topics that represents what the event mention is referring to. This paper is focused on temporal information processing and topic-based semantic representation.

\subsection{Temporal Information Processing}

The TimeML (Saurí et al., 2006) annotation scheme has now been adopted as a standard by a large number of researchers in the field of temporal information annotation. It represents not only events and temporal expressions, but also links (Pustejovsky et al., 2003)

A manual annotation of event mentions and the DCT of texts have been considered as an input of the system, and an automatic system has been used to perform the annotation with temporal expressions and temporal links in order to be able to establish a complete timeline of the input texts. If a plain text is considered, systems such TIPSem (Temporal Information Processing using Semantics) (Llorens et al., 2013; Llorens et al., 2012) ${ }^{1}$ are able to automatically annotate all the temporal expressions (TIMEX3), events (EVENT) and links between them.

Once the temporal links have been established, all the specific temporal information for each event is inferred by means of temporal links navigation. This information allows us to determine temporal compatibility between all the events considered.

\subsection{Topic-based Semantic Representation}

The meaning of each event structure has been represented by using Topic Modeling (Blei, 2012) on a reference corpus. Topic modeling is a family of algorithms that automatically discover topics from a collection of documents. More specifically, we apply the Latent Dirichlet Allocation (LDA) (Blei et al., 2003), which follows a bottom up approach. Each word is assigned to a topic according to the co-ocurrence words in the context (document) and the topics assigned to this word in other documents. In formal terms, a topic is a distribution on a fixed vocabulary.

We have applied the LDA to the WikiNews corpus. ${ }^{2}$ Each topic in this corpus is represented using the twenty most prominent words.

\section{Architecture of the System}

Our approach to build timelines from written news in English implies event coreference resolution by applying three cluster processes in sequential order: a temporal cluster, a lemma cluster, and a topic cluster. It combines various resources:

- Named entity recognition, using OpeNER web services. $^{3}$

- TimeML automatic annotation of texts using TipSEM system (Llorens et al., 2010).

- The NLTK ${ }^{4}$ verb lemmatizer based on WordNet (Fellbaum, 1998).

- The SENNA (Collobert et al., 2011) Semantic Roles Labeling.

\footnotetext{
${ }^{1}$ http://gplsi.dlsi.ua.es/demos/TIMEE/

${ }^{2}$ https://dumps.wikimedia.org/enwikinews /

${ }^{3}$ http: //www. opener-project.eu/

webservices /

${ }^{4}$ http: //www.nltk.org/
} 
- The LDA Topic Modeling algorithm, using MALLET (McCallum, 2002).

\subsection{Target Entity Filtering}

If the target entity filtering is to be performed then it is first necessary to resolve the named entity recognition and coreference resolution. This is done by integrating the external OpeNER web services into our proposal. More specifically, the components applied in our proposal are the NER component, ${ }^{5}$ which identifies the names of people, cities, and museums, and classifies them in a semantic class (PERSON, LOCATION, etc.) and the coreference resolution component, ${ }^{6}$ whose objective is to identify all those words that refers to the same object or entity.

Only those events that are part of sentences containing the target entity or a coreference entity of the target will be selected for the final timeline.

\subsection{Temporal Clustering Approach}

A plain text was considered and we use the TIPSem system to automatically annotate all the temporal expressions (TIMEX3), events (EVENT) and links between them. The TLINKS annotated in the text are used in order to extract the time context of each event and make it possible to infer both time at which each event occurs and the temporal ordering between the events in the text. Moreover, if we are able to determine the time of the event, we will be able to determine temporal compatibility between events, even when they are contained in different documents, thus signifying that crossdocument event coreference resolution is also possible.

In this first step, all the events from the different documents that occurring on the same date will therefore be part of the same cluster. The clusters are positioned in ascending ordered based on the date assigned.

\subsection{Semantic Clustering Based on Lemmas}

Once all the events that share temporal information and the target entity have been grouped together, we apply a simple clustering based on head word lemmas. This lemma-based clustering groups together all event mentions with the same head word lemma,

\footnotetext{
${ }^{5}$ http://opener.olery.com/ner

${ }^{6}$ http://opener.olery.com/coreference
}

the same temporal information and the same target entity. We therefore assume that all these event mentions corefer to the same event. This is our Run 1 at the competition.

\subsection{Semantic Clustering Based on Topics}

The problem of the lemma-based cluster is that it does not take into account the argument structure of the event. This last clustering therefore attempts to solve this problem by extracting the semantic roles from each event and representing their meaning by using topics on a reference corpus. This approach has three steps:

1. Using SENNA (Collobert et al., 2011) as Semantic Roles Labeling, we have detected roles $\mathrm{A} 0$ and $\mathrm{A} 1{ }^{7}$ which are related to the event mention head word. For each role we extract only the nouns.

2. We have extracted 500 topics from WikiNews using Topic Modeling with MALLET. All these topics are used as a knowledge base. We will use only the most representative words for each topic (the twenty words with the greatest weight) and the weights that they have in each topic.

3. Finally, we have created an event-topic matrix. Each event (raws) is represented by a vector. The values of the vector are the addition of weights of each argument noun in each topic (columns).

For example, if the nouns in arguments A0 and A1 are "users, problems, phones", we represent their meanings according to the topics $t_{n}$ assigned to them by applying LDA to WikiNews ( user $=t_{0}, t_{3}, t_{5}$, problems $=t_{0}, t_{2}$, phones $=t_{5}, t_{6}$, etc). Then, the event $e$ of this sentence is represented by a $n$ dimensional vector in which $n$ is the amount of topics (500) and whoses values are the addition of weight of each noun in each topic $T_{n}$.

In order to group together similar event mentions, we have applied a k-means clustering algorithm to these event vectors. ${ }^{8}$ The distance metric used has

\footnotetext{
${ }^{7}$ In order to represent Semantic Roles, SENNA uses the tag set proposed by Proposition Bank Project (http://verbs . colorado.edu/ mpalmer/projects/ace.html) A0 and $\mathrm{A} 1$ represent the main roles related to each verb.

${ }^{8}$ Note that it has been applied only to the events previously clustered following the lema-based approach (Run 1).
} 
been Euclidean Distance. The number of cluster has been adjusted to two. ${ }^{9}$ Therefore, each cluster with the same head word lemma, the same temporal information and the same target entity is then reclustered according to the similarity of the main topics of its arguments. This cluster corresponds to our Run 2 at the competition.

\section{Evaluation Results}

SemEval-2015 Task 4 consists on building timelines from written news in English in which a target entity is involved. The input data provided by the organizers is therefore a set of documents and a set of target entities related to those documents. Two different tracks are proposed in the task, along with their subtracks:

- Track A: This consists of using raw texts as input and obtaining full timelines. Subtrack A has the same input data, but the output will be the timeLines of only ordered events (no assignment of time anchors).

- Track B: This consists of using texts with manual annotation of events mentions as input data. Subtrack B has the same input data but the output will be timeLines of only ordered events.

In the Semeval-2015 Task 4 competition we have participated in Track B and Subtrack B. The results for the Micro-average F-score measure obtained by our approach in the competition are shown in Table 1.

\begin{tabular}{lcccc}
\hline TRACK & Corpus1 & Corpus2 & Corpus3 & Total \\
\hline TrackB-R1 & 22.35 & 19.28 & 33.59 & $\mathbf{2 5 . 3 6}$ \\
TrackB-R2 & 20.47 & 16.17 & 29.90 & $\mathbf{2 2 . 6 6}$ \\
SubTrackB-R1 & 18.35 & 20.48 & 32.08 & $\mathbf{2 3 . 1 5}$ \\
SubTrackB-R2 & 15.93 & 14.44 & 27.48 & $\mathbf{1 9 . 1 8}$ \\
\hline
\end{tabular}

Table 1: Results for GPLSIUA Approach.

Although the Micro-FScore results are not very high, the results obtained by our approach are the highest in all of the corpus evaluated by the organizers. Our approach obtained an improvement of $7 \%$ compared with the other participant in Track B and a $6.48 \%$ in Subtrack B.

\footnotetext{
${ }^{9} \mathrm{We}$ have used PyCluster tool: https://pypi. python.org/pypi/Pycluster
}

\section{Conclusions}

The results show that our approach is suitable for the task in hand. On the one hand, temporal information is automatically extracted with a temporal information processing system which makes it possible to infer and determine the time at which each event has occurred. On the other hand, the semantic similarity based on the verb is sufficient to group together coreferent events.

The basic method (Run 1), consisting of searching for similar verb lemma, eventually proved to be the best. We have therefore carried out an in-depth analysis of the results obtained for Run 2 and have observed three main drawbacks in the Topic Modeling approach:

- The K-means algorithm forces us to fix the number of clusters beforehand, and this has been fixed at 2. However, there is often only one correct cluster. Another approach without a fixed number of topics will improve the approach. Bejan and Harabagiu (2014), for example, suggest inferring this value from data.

- The representativity of each event mention depends directly on the amount of topics extracted from the reference corpus. Many topics will produce excessive granularity, and few topics will be unrepresentative. We have set the number of topics at 500, but it is necessary to study whether another amount of topics will improve the results.

- This approach depends excessively on the representativity of the reference corpus. We believe using larger corpora should improve the results.

As Future work, we plan to use other similarity measures and clustering algorithms in an attempt to solve the problem of previously fixed number of clusters. We also plan to evaluate using different Topic Modeling configurations.

\section{Acknowledgments}

We would like to thank the anonymous reviewers for their helpful suggestions and comments. Paper partially supported by the following projects: ATTOS (TIN2012-38536-C03-03), LEGOLANG-UAGE (TIN2012-31224), SAM (FP7-611312), FIRST (FP7-287607) DIIM2.0 (PROMETEOII/2014/001) 


\section{References}

Cosmin Adrian Bejan and Sanda Harabagiu. 2014. Unsupervised Event Coreference Resolution. Computational Linguistics, 40(2):311-347.

David M Blei, Andrew Y Ng, and Michael I Jordan. 2003. Latent Dirichlet Allocation. Journal of Machine Learning Research, 3:993-1022.

David M Blei. 2012. Probabilistic Topic Models. Communications of the ACM, 55(4):77-84.

Ronan Collobert, Jason Weston, Léon Bottou, Michael Karlenand Koray Kavukcuoglu, and Pavel Kuksa. 2011. Natural Language Processing (Almost) from Scratch. Journal of Machine Learning Research, pages $41-71$.

Agata Cybulska and Piek Vossen. 2013. Semantic relations between events and their time, locations and participants for event coreference resolution. In RANLP.

Christiane Fellbaum. 1998. WordNet: An Electronic Lexical Database (Language, Speech, and Communication). MIT Press.

Malka Rappaport Hovav, Edit Doron, and Ivy Sichel. 2010. Lexical Semantics, Syntax, and Event Structure. Oxford University Press, Oxford.

Beth Levin and Malka Rappaport-Hovav. 2005. Argument realization. Cambridge University Press, Cambridge.

Hector Llorens, Estela Saquete, and Borja NavarroColorado. 2010. TIPSem (English and Spanish): Evaluating CRFs and Semantic Roles in TempEval-2. In Proceedings of the 5th International Workshop on Semantic Evaluation.

Hector Llorens, Estela Saquete, and Borja NavarroColorado. 2012. Automatic System for Identifying and Categorizing Temporal Relations in Natural Language. International Journal of Intelligent Systems, 27(7):680-703.

Hector Llorens, Estela Saquete, and Borja NavarroColorado. 2013. Applying Semantic Knowledge to the Automatic Processing of Temporal Expressions and Events in Natural Language. Information Processing \& Management, 49(1):179-197.

Andrew Kachites McCallum. 2002. Mallet: A machine learning for language toolkit. http://mallet.cs.umass.edu.

James Pustejovsky, José M. Castaño, Robert Ingria, Roser Saurí, Robert Gaizauskas, Andrea Setzer, and Graham Katz. 2003. TimeML: Robust Specification of Event and Temporal Expressions in Text. In IWCS5 .

Roser Saurí, Jessica Littman, Robert Knippen, Robert Gaizauskas, Andrea Setzer, and James Pustejovsky, 2006. TimeML Annotation Guidelines 1.2.1 (http://www.timeml.org/). 